First Peoples Child \& Family Review

An Interdisciplinary Journal Honouring the Voices, Perspectives, and Knowledges of First Peoples through Research, Critical Analyses, Stories, Standpoints and Media Reviews

\title{
Influence de l'estime de soi, des qualités relationnelles parents-enfants, du soutien social et de l'agression sexuelle sur la résilience auprès d'adolescents autochtones et caucasiens
}

\author{
François Muckle, Jacinthe Dion, Isabelle Daigneault, Amélie Ross and Pierre \\ McDuff
}

Volume 7, Number 1, 2012

URI: https://id.erudit.org/iderudit/1068868ar

DOI: https://doi.org/10.7202/1068868ar

\section{See table of contents}

Publisher(s)

First Nations Child and Family Caring Society of Canada

\section{ISSN}

1708-489X (print)

2293-6610 (digital)

\section{Explore this journal}

\section{Cite this article}

Muckle, F., Dion, J., Daigneault, I., Ross, A. \& McDuff, P. (2012). Influence de l'estime de soi, des qualités relationnelles parents-enfants, du soutien social et de l'agression sexuelle sur la résilience auprès d'adolescents autochtones et caucasiens. First Peoples Child \& Family Review, 7(1), 99-117.

https://doi.org/10.7202/1068868ar

\section{Article abstract}

Cet article a pour objectif d'explorer la résilience psychologique auprès d'adolescents caucasiens et autochtones. Bien que plusieurs études aient été réalisées sur cette thématique auprès des caucasiens, peu ont été conduites auprès des peuples des Premières Nations, bien qu'ils aient vécu plusieurs événements traumatiques depuis la colonisation. Pour ce faire, 227 participants autochtones et caucasiens âgés de 14 à 17 ans ont répondu à des questionnaires autorapportés. La résilience a été conceptualisée en termes d'absence de détresse psychologique et fut évaluée par le score total obtenu au Trauma Symptom Checklist for Children (TSC-C; Briere, 1989). Le niveau d'estime de soi des adolescents fut mesuré, de même que des facteurs interpersonnels tels le soutien parental, social et communautaire; afin d'être cohérent avec une perspective autochtone qui repose sur une vision interrelationnelle entre l'individu et sa communauté. Dans l'ensemble, les résultats de cette étude révèlent que les adolescents autochtones n'ont pas été davantage victimes d'agression sexuelle que leurs homologues caucasiens, mais ils ont vécu davantage d'événements de vie délétères. Les résultats de l'analyse de régression suggèrent que l'estime de soi et la capacité de l'adolescent à rechercher de l'aide dans sa communauté sont associées à moins de détresse psychologique, soit plus de résilience. Toutefois, avoir subi une agression sexuelle, avoir été exposé à plusieurs évènements de vie stressants, être de sexe masculin et être un adolescent non-autochtone est relié à plus de détresse psychologique. En somme, les présents résultats suggèrent l'importance d'utiliser une approche écologique qui implique à la fois les facteurs personnels et communautaires dans la compréhension des facteurs de résilience.
Copyright ( François Muckle, Jacinthe Dion, Isabelle Daigneault, Amélie Ross, Pierre McDuff, 2012
This document is protected by copyright law. Use of the services of Érudit (including reproduction) is subject to its terms and conditions, which can be viewed online.

https://apropos.erudit.org/en/users/policy-on-use/ 


\title{
First Peoples Child \& Family Review
}

An Interdisciplinary Journal Honoring the Voices, Perspectives and Knowledges of First Peoples through Research, Critical Analyses, Stories, Standpoints and Media Reviews

\section{Influence de l'estime de soi, des qualités relationnelles parents-enfants, du soutien social et de l'agression sexuelle sur la résilience auprès d'adolescents autochtones et caucasiens ${ }^{1}$}

\author{
François Muckle ${ }^{\mathrm{ab}}$, Jacinthe Dion ${ }^{\mathrm{ab}}$, Isabelle Daigneault ${ }^{\mathrm{bc}}$, \\ Amélie Ross ${ }^{a b}$, et Pierre McDuffbc
}

\footnotetext{
a Département des sciences de la santé, Université du Québec à Chicoutimi, Chicoutimi, Québec, Canada.

${ }^{b}$ Centre de recherche sur les problèmes conjugaux et les agressions sexuelles (CRIPCAS).

${ }^{\mathrm{c}}$ Département de psychologie, Université de Montréal, Montréal, Québec, Canada.
}

\begin{abstract}
Les auteurs souhaitent remercier la direction de l'école concernée, M. Bouchard, pour sa précieuse collaboration à ce projet ainsi que tous les participants qui ont accepté de partager plusieurs aspects de leur vie personnelle (Tshinashkumitin). Un remerciement spécial à Marie-Frédérique Bouchard pour avoir facilité les contacts avec le milieu de recherche, pour la mise en page du questionnaire et pour l'entrée de données, également effectuée avec la collaboration de Delphine Lagacé. Cette étude a bénéficié d'une subvention du Programme d'aide institutionnelle à la recherche de l'Université du Québec à Chicoutimi, de même qu'elle s'inscrit dans le cadre des activités de recherche du Centre de recherche sur les problèmes conjugaux et les agressions sexuelles (CRIPCAS).
\end{abstract}

\section{Résumé}

Cet article a pour objectif d'explorer la résilience psychologique auprès d'adolescents caucasiens et autochtones. Bien que plusieurs études aient été réalisées sur cette thématique auprès des caucasiens, peu ont été conduites auprès des peuples des Premières Nations, bien qu'ils aient vécu plusieurs événements traumatiques depuis la colonisation. Pour ce faire, 227 participants autochtones et caucasiens âgés de 14 à 17 ans ont répondu à des questionnaires autorapportés. La résilience a été conceptualisée en termes d'absence de détresse psychologique et fut évaluée par le score total obtenu au Trauma Symptom Checklist for Children (TSC-C; Briere, 1989). Le niveau d'estime de soi des adolescents fut mesuré, de même que des facteurs interpersonnels tels le soutien parental, social et communautaire; afin d'être cohérent avec une perspective autochtone qui repose sur une vision interrelationnelle entre l'individu et sa communauté. Dans l'ensemble, les résultats de cette étude révèlent que les adolescents autochtones n'ont pas été davantage victimes d'agression sexuelle que leurs homologues caucasiens, mais ils ont vécu davantage d'événements de vie délétères. Les résultats de l'analyse de régression suggèrent que l'estime de soi et la capacité de l'adolescent à rechercher de l'aide dans sa communauté sont associées à moins de détresse psychologique, soit plus de résilience. Toutefois, avoir subi une agression sexuelle, avoir été exposé à plusieurs évènements de vie stressants, être de sexe

Corresponding author:

Jacinthe Dion, Professeure en psychologie, Départment des sciences de la santé, Université du Québec á Chicoutimi, 555 boul. de l'Université, Chicoutimi (QC) G7H 2B1 Jacinthe_Dion@uqac.ca; (418) 545-5011, ext 5663, (418) 545-5011, poste 566w3 masculin et être un adolescent non-autochtone est relié à plus de détresse psychologique. En somme, les présents résultats suggèrent l'importance d'utiliser une approche écologique qui implique à la fois les facteurs personnels et communautaires dans la compréhension des facteurs de résilience.

Mots clés : Résilience, symptômes, facteurs de risque, facteurs de protection, adolescents, Autochtone, agression sexuelle, estime de soi, soutien communautaire, soutien familial.

1 Influence of self-esteem, parent-child relationships, social support and sexual abuse on resilience among Aboriginal and Caucasian adolescents 


\section{Influence de l'estime de soi, des qualités relationnelles parents-enfants ...}

\section{Abstract}

This article aims to explore resilience among Caucasian and Aboriginal adolescents. Although several studies have been conducted on this topic among Caucasians, few concern First Nation peoples, even though the latter have experienced many traumatic events since colonization. A total of 227 Aboriginal and Caucasian participants aged between 14 and 17 years completed questionnaires. Resilience has been conceptualized as the absence of psychological distress and was evaluated by the total score obtained on the Trauma Symptom Checklist for Children. The level of self-esteem in adolescents was measured, as well as interpersonal factors such as parental, social and community support, in order to be consistent with an Aboriginal perspective based on an interrelational view between the individual and his community. Overall, the results of this study indicate that sexual abuse is not more common among Aboriginal youth than their Caucasian counterparts, but they do experience more adverse life events. The results of regression analyses suggest that self-esteem and the capacity of adolescents to seek help in the community are associated with less psychological distress, and thus, more resilience. However, having been sexually abused, being exposed to more stressful life events, being male and being a Caucasian teenager are all factors related to greater psychological distress. Overall, these results suggest the importance of utilizing an ecological approach that involves both personal and community factors in understanding resilience factors.

Key words: Resilience, symptoms, risk factors, protective factors, adolescents, Aboriginal, sexual abuse, selfesteem, community support, family support.

\section{Introduction}

En dépit des conséquences néfastes de l’agression sexuelle, il semble que certaines personnes ne présentent en apparence que peu de séquelles à la suite des agressions sexuelles dont elles ont été l'objet. En effet, on estime que près de 30 \% des personnes ayant subi une agression sexuelle ne souffriront d'aucun symptôme (Kendall-Tackett, Williams, \& Finkelhor, 1993). Cette constatation a porté certains chercheurs à s'intéresser aux facteurs reliés à la résilience chez ces personnes (Daigneault, Hébert, \& Tourigny, 2007; DuMont, Widom, \& Czaja, 2007; LaFromboise, Hoyt, Oliver, \& Whitbeck, 2006; Zahradnik, Stewart, O’Connor, Stevens, Ungar, \& Wekerle, 2010). À ce jour, il n'y a toutefois pas d'études s'étant intéressées à cette question auprès des adolescents autochtones vivant au Québec, et ce, malgré un niveau de violence sexuelle significatif au sein des Premières Nations (Collin-Vezina, Dion, \& Trocme, 2009), possiblement en conséquence aux nombreux évènements traumatiques survenus depuis la colonisation (Muckle \& Dion, 2008). Une meilleure connaissance des facteurs de risque et de protection favorisant ou entravant la résilience pourra éventuellement permettre de répondre plus adéquatement aux besoins des enfants et des familles tant chez les caucasiens que chez les peuples autochtones. Le présent article se propose donc d'explorer les facteurs reliés à la résilience psychologique en contexte d'agression sexuelle auprès d'adolescents autochtones et caucasiens.

\section{Définition, prévalence et incidence de l'agression sexuelle}

L'agression sexuelle envers un enfant ou un adolescent concerne toute activité sexuelle perpétrée sous la menace, par la force, l'intimidation ou la manipulation (ministère de la Justice du Canada, 2006a). Elle est habituellement le fait d'un individu plus âgé que la victime, qui utilise la menace physique et psychologique afin de maintenir son emprise (ministère de la Justice du Canada, 2006b). Lagression sexuelle prend diverses formes, il peut s'agir de relations sexuelles, de tentatives d'avoir une relation sexuelle, d'attouchements aux organes génitaux, de l'exhibitionnisme, de l'exploitation sexuelle telles la pornographie et la prostitution, du harcèlement sexuel et enfin du voyeurisme (Centre national d'information sur la violence dans la famille, 2006; Trocmé \& Wolfe, 2001). 
First Peoples Child \& Family Review, Volume 7, Number 1, 2012

Deux études québécoises et deux méta-analyses récentes indiquent que la prévalence de l'agression sexuelle dans l'enfance se situe entre 8 et $10 \%$ pour les hommes et entre 18 et $22 \%$ chez les femmes (Pereda, Guilera, Forns, \& Gòmez-Benito, 2009; Stoltenborgh, van Ijzendoorn, Euserl, \& Bakermans-Kranenburg, 2011; Tourigny, Gagné, Joly, \& Chartrand, 2006; Touringy, Hébert, Joly, Cyr, \& Baril, 2008). Chez les populations autochtones, la prévalence est plus difficile à établir. En dépit du fait que plusieurs communautés autochtones parlent aujourd'hui davantage de la problématique de l'agression sexuelle, il s'agit encore d'un tabou important qui a des répercussions sur le nombre de dévoilements, ce qui rend la prévalence de la problématique difficile à établir (Muckle \& Dion, 2008). De plus, certains résultats d'études ont été mal interprétés ou mal cités, ce qui a conduit plusieurs à croire que la prévalence de l'agression sexuelle pouvait se situer autour de 75 à $80 \%$ chez les personnes autochtones (voir Collin-Vézina, et al., 2009). Par exemple, les résultats de Leclair et al. (1996) ont montré que $75 \%$ des victimes autochtones de violence sexuelle étaient des filles âgées de moins de 18 ans et non que $75 \%$ des filles autochtones avaient été victimes d'agression sexuelle (tel que rapporté par Jiwani et al. [1999] qui cite incorrectement McIvor \& Nahanee [1998] concernant l'étude de Leclair et al. [1996]). De même, à partir d'entrevues conduites auprès de plusieurs acteurs, tels que les victimes, agresseurs, intervenants sociaux, aînés, etc., Levan (1989) déduit que jusqu'à 80 \% des filles autochtones de l'Arctique ont été victimes d'agression sexuelle. Bien que ce taux n'ait jamais prétendu être une véritable estimation de la prévalence de l'agression sexuelle, il a ensuite été repris par plusieurs auteurs (p. ex. : Commission royale sur les peuples autochtones, 1996). Ainsi, les résultats d'une récente synthèse des écrits indiquent qu'il est sans doute plus réaliste d'estimer qu'entre 25 et $50 \%$ des adultes autochtones ont été agressés sexuellement avant l'atteinte de leur majorité (CollinVézina et al., 2009).

\section{Impacts de l'agression sexuelle}

Lagression sexuelle a des répercussions importantes et est associée, selon les cas, à des épisodes dépressifs, de l'anxiété, une dépendance à l'alcool, des comportements antisociaux, un syndrome de stress post-traumatique, des comportements autodestructeurs, une faible estime de soi, des difficultés à faire confiance aux autres, et enfin, les personnes peuvent être " revictimisées " (Finkelhor, 1990; Kendall-Tackett et al., 1993; Lynskey \& Fergusson, 1997; Macmillan et al., 2001; Maniglio, 2009; Mullen, Martin, Anderson, Romans, \& Herbison, 1996; Putnam, 2003; VeitchWolfe, Gentile, \& Wolfe, 1989). Notons que des chercheurs observent le même type de séquelles chez les personnes autochtones (Centre des Premières Nations et Organisation nationale de la santé autochtone [ONSA], 2006; Corrado \& Cohen, 2003; Yellow Horse Brave Heart, \& DeBruyn, 1998). De plus, les femmes autochtones victimes d'agression sexuelle présenteraient significativement plus de symptômes somatiques, de troubles du sommeil et de problèmes sexuels que leurs homologues caucasiennes (Barker-Collo, 1999). En outre, les résultats d'une étude qualitative réalisée auprès de femmes autochtones ayant été agressées dans l'enfance révèlent que les victimes éprouvent de la culpabilité, un fort sentiment de vulnérabilité, des épisodes dissociatifs, et ont tendance à s'autodéprécier et à avoir honte de leur identité autochtone (McEvoy \& Daniluk, 1995).

À la lumière des éléments précités, il apparaît que l'agression sexuelle est un traumatisme pouvant produire des séquelles multiples chez les gens qui en sont la cible. Néanmoins, ces personnes n'empruntent pas toutes la même trajectoire; certaines paraissant s'en remettre mieux que d'autres; elles ont la capacité d'être résilientes. 


\section{La résilience}

Bien que la résilience soit définie et opérationnalisée différemment selon les études (Daigneault, Cyr, \& Tourigny, 2007; Kolar, 2011), deux grandes catégories de définition semblent s'y retrouver: l'une mettant l'accent sur les dimensions psychologiques de l'individu et l'autre y intégrant les interactions de la personne avec son environnement (Muckle \& Dion, 2008). Cette dernière catégorie semble pouvoir intégrer le construit de résilience en contexte multiculturel puisquelle considère l'interaction des facteurs de risque et de protection selon le contexte et la culture.

Peu de recherches sur la résilience ont été réalisées chez les jeunes autochtones. Mentionnons cependant létude de LaFromboise et ses collègues (2006) qui s'est intéressée aux facteurs de résilience chez 212 enfants et adolescents âgés de 10 à 15 ans. La résilience a été mesurée comme un construit multidimensionnel, soit la présence de comportements scolaires pro-sociaux, tels que les attitudes envers l'école, les notes scolaires et l'absence de problèmes de comportements, tels que la consommation d'alcool et de drogues, les comportements externalisés en présence d'adversité à la maison (p. ex. : pauvreté, problèmes de toxicomanie chez les parents). Le sentiment d'appartenance culturelle, la chaleur maternelle, ne pas percevoir de discrimination et le soutien communautaire se sont avérés des facteurs significativement associés à la résilience chez les 126 jeunes vivant en contexte défavorable. Une autre étude portant sur le soutien communautaire et impliquant deux communautés autochtones américaines révèle que ce facteur de protection peut avoir un impact important chez les parents ayant été victimes d'agression sexuelle dans l'enfance, en leur permettant d'éviter qu'ils perpétuent les situations d'abus envers leurs propres enfants (Libby, Orton, Beals, Buchwald, \& Manson, 2008). Par ailleurs, il appert que les élèves d'une communauté Mi'Kmaq qui ont été exposé à de la violence, mais qui ont un sens de coopération, de la persévérance, des relations positives avec leur famille et leurs amis, de même qu'une implication dans la communauté, vont vivre moins de symptômes d'intrusion (p. ex. : cauchemars) associés au trouble de stress post-traumatique (Zahradnik et al., 2011).

Ainsi, la résilience n'est pas le fait d'individus invulnérables et ne veut pas dire que les habiletés ayant permis de surmonter avec succès un type de traumatisme seraient généralisables, pour une même personne, à tout autre type de traumatisme (Lemay, 2001). La résilience est davantage envisagée tel un processus, une capacité permettant une adaptation adéquate en dépit de circonstances menaçantes ou d'évènements de vie difficiles (Luthar, Cicchetti, \& Becker, 2000; Kolar, 2011). Elle peut également conduire à un potentiel de protection susceptible dêtre mobilisé de nouveau (Anaut, 2002).

Depuis, un modèle de la résilience basé sur les facteurs de protection avance que ceux-ci interagissent avec les facteurs de risques pour diminuer les effets nocifs du stress, ce qui aurait un impact significatif quant aux fonctions adaptatives de l'individu (Golse, 2006). Il apparait donc que la résilience implique une interaction protectrice multifactorielle favorisant l'adaptation, et qu'elle est le résultat d'une co-construction, dans la mesure où elle s'inscrit à travers un échange constant entre l'individu et son environnement, et ce, tout au long de son développement.

\section{Facteurs de protection et résilience}

Maintes recherches ont tenté d'identifier les facteurs de protection les plus susceptibles de contribuer à la résilience (Constantine, Benard, \& Diaz, 1999; Daigneault et al., 2007; DuMont et al., 2007). Il appert qu'ils sont multiples et impliquent tant l'individu, la famille que la communauté (Drapeau, Saint-Jacques, Lépine, Bégin, \& Bernard, 2007; Wolkow \& Fergusson, 2001). Parmi ces 
First Peoples Child \& Family Review, Volume 7, Number 1, 2012

facteurs, trois ont été retenus dans le cadre de la présente étude, soit l'estime de soi, la qualité des relations parents-enfants perçue par l'adolescent et la capacité à aller chercher de l'aide dans la communauté. Ce choix est cohérent avec le construit de la résilience qui implique non seulement des facteurs intra-individuels, mais plus largement l'étude des aspects relationnels susceptibles de contribuer à celle-ci. En outre, la dimension communautaire a été intégrée puisqu’elle représente un aspect important dans l'étude de la résilience en contexte multiculturel (Tummala-Narra, 2007).

\section{Estime de soi.}

Lestime de soi réfère à la perception qu'une personne entretient quant à sa valeur personnelle (Harter, Whitesell, \& Junkin, 1998). Elle se construit à travers certaines relations, dans divers contextes (Harter, Waters, \& Whitesell, 1998) et selon un processus dynamique et continu (Jendoubi, 2002). Une estime de soi positive a notamment été associée au bien-être psychologique (Rosenberg, Schoeler, Schoebach, \& Rosenberg, 1995) en plus de favoriser les interactions sociales (Chen Yi-Feng, Huang, \& Tjosvold, 2008). De même, elle contribue à une plus grande sécurité émotionnelle, une tolérance accrue aux frustrations et permet l'établissement d'un regard plus juste en ce qui concerne les aptitudes et qualités d'un individu (Fortin, 1989).

En outre, elle constitue un facteur de protection en regard de la psychopathologie chez l'adolescent (MacDonald \& O'Hara, 1996). À ce titre, l'estime de soi s'est avérée être une caractéristique significative des individus affichant davantage de résilience en contexte de maltraitance (Scott Heller, Larrieu, D’imperio, \& Boris, 1999), mais non en contexte de vie difficile chez de jeunes autochtones (Lafromboise et al., 2006). Une recherche longitudinale impliquant 16 adolescentes agressées sexuellement a aussi révélé que l'estime de soi et la cohésion interne des participants s'amélioraient au cours d'une année, tandis qu’en parallèle leurs symptômes diminuaient (Daigneault et al., 2007).

\section{Qualité des relations parents-enfants.}

Il semble également qu'un milieu familial soutenant soit vecteur de résilience (Pinkerton \& Dolan, 2007). En outre, il permet le développement d'un bien-être psychologique accru en diminuant le risque de troubles psychiatriques (Carlton et al., 2006). En effet, le fonctionnement familial, plus particulièrement la qualité des relations parents-enfants, exerce un effet significatif à long terme sur les capacités d'adaptation et donc de résilience chez les victimes d'agression sexuelle en venant moduler déventuelles psychopathologies ou problèmes d'ajustement (Lynskey \& Fergusson, 1997). Chez des survivantes d'agression sexuelle, le fonctionnement familial exerce un effet notable sur le sentiment de compétence personnelle dans la gestion du quotidien, sur la qualité des relations interpersonnelles et l'acceptation de soi. Ainsi, la cohésion familiale, soit le soutien parental, la sensibilité et la constance, de même que la présence d'un climat familial conflictuel sont reliées de façon positive et négative, respectivement, au bien-être des participantes (McClure, Chavez, Agars, Peacock, \& Matosian, 2008). Ajoutons qu'une étude rétrospective américaine (DuMont et al., 2007) portant sur un échantillon de 676 dossiers d'enfants victimes de maltraitance montre que les victimes ayant grandi dans des conditions familiales stables (avec les deux parents ou lors d'un placement de longue durée) sont significativement plus résilientes à l'adolescence que celles ayant été placées à l'extérieur de leur famille pendant un court laps de temps.

(c) François Muckle, Jacinthe Dion, Isabelle Daigneault, Amélie Ross, et Pierre McDuff 


\section{Soutien social et communautaire.}

Le soutien communautaire est conçu comme un aspect favorisant l'empowerment (le pouvoir de changement) chez l'individu, ce qui est susceptible de favoriser la résilience. Daigneault et ses collaborateurs (2007) avancent à cet effet que les communautés peuvent être des vecteurs de résilience auprès des victimes d'agression sexuelle à travers la mise en place d'interventions thérapeutiques axées sur le développement de la confiance en soi et du pouvoir de changement. Le soutien communautaire ou social (infrastructures, pairs) protège la personne, car il joue un rôle tampon entre l'événement stressant et ses effets délétères et son expression en termes de santé mentale (Armstrong, Birnie-Lefcovitch, \& Ungar, 2005). Par exemple, la présence d'établissements, comme une école ou un centre de loisirs communautaire, où l'on développe les compétences, un sentiment d'appartenance et la détermination sont des facteurs de résilience (Drapeau et al., 2007). De même, le fait pour un adolescent de s'impliquer au sein de sa communauté contribue au développement de ses compétences sociales, de ses capacités de résolution de problème, de son autonomie et de liens significatifs (Kegler et al., 2005; Scales, Benson, Leffert, \& Blyth, 2000).

\section{Objectifs de la présente étude}

La notion de résilience chez les jeunes victimes d’agression sexuelle ne bénéficie que d’un nombre limité de recherches, bien quelle soit sujette à un intérêt croissant (Drapeau et al., 2007). De surcroît, le peu détudes concernant les adolescents autochtones mène à souligner l'importance de travailler à identifier les facteurs de protection qui favorisent la résilience chez ceux ayant été potentiellement victimes d’agression sexuelle. La présente étude a été élaborée en tenant compte de l'histoire et des politiques colonialistes à l'égard des membres des Premières Nations et en collaboration avec des intervenants autochtones, dans le respect de la culture et des traditions autochtones.

L'objectif de la présente étude est d'évaluer l'influence de l'estime de soi, de la qualité de la relation parent-adolescent et de la capacité à chercher de l'aide dans sa communauté sur la résilience psychologique. Notons que l'absence de symptomatologie psychologique sera considérée tel un indicateur de résilience dans la présente étude. Plus précisément, moins l'adolescent présente de symptômes psychologiques, plus il est considéré résilient. En se fondant sur les données trouvées dans la littérature, il est attendu que 1) plus le niveau d'estime de soi augmente, plus la résilience augmente; 2) plus la qualité de la relation de l'adolescent avec ses parents est élevée, plus la résilience augmente; 3) plus l'adolescent a la capacité d'aller chercher ou de recevoir du soutien (social, à l'école, ou communautaire) plus la résilience augmente; et enfin, 4) que les adolescents autochtones auront subi davantage d'agressions sexuelles, et 5) qu'ils auront été exposés à davantage d'événements de vie stressants.

\section{Méthode}

\section{Participants et déroulement}

La présente étude a été réalisée en avril 2010 auprès de 227 adolescents autochtones et caucasiens habitant au Québec et fréquentant un établissement d'enseignement secondaire situé en milieu défavorisé (MELS, 2011). Huit participants ont été exclus de léchantillon, car leurs questionnaires comprenaient un nombre élevé de données manquantes. Les participants ont été recrutés par l'intermédiaire de leurs professeurs après avoir reçu au préalable l'autorisation de la direction d'école concernée et une certification éthique. Chaque élève des classes rencontrées a reçu un 
First Peoples Child \& Family Review, Volume 7, Number 1, 2012

questionnaire contenu dans une enveloppe afin d'assurer la confidentialité. Un formulaire de consentement leur a été remis, leur expliquant les modalités de la recherche, ce qui leur a permis de faire un choix libre et éclairé. La collecte de données était entièrement anonyme et ne permettait ainsi en aucun moment l'identification des répondants. Les participants lisaient et parlaient tous le français. La collecte de données sest déroulée dans la bibliothèque de létablissement où deux groupes d'élèves se sont présentés à tour de rôle et ont effectué la passation d'une durée approximative de 30 minutes. Des assistants de recherche, ainsi que les responsables de létude, étaient disponibles afin de répondre à leurs questions et s'assuraient qu'ils ne parlent pas entre eux.

Léchantillon autochtone était constitué de 61 adolescents ( $27 \%$; $46 \%$ de filles). Lâge se situait entre 14 et 17 ans (moyenne d'âge, 15,44 ans, ÉT $=1,02$ ) et $85 \%$ vivaient avec un de leurs parents ou avec les deux. Lensemble des cycles secondaires a été représenté avec en plus près de $20 \%$ étaient en cheminement particulier. Léchantillon caucasien était quant à lui constitué de 166 adolescents ( $73 \%$; $48 \%$ de filles) âgés de 14 à 17 ans (moyenne d’âge 15,98 ans, ÉT=0,74) et dont 99 \% vivaient avec l'un de leurs parents ou chez les deux. L'ensemble des cycles secondaires était représenté et aucun nétait en cheminement particulier.

\section{Instruments de mesure utilisés}

Six instruments de mesure furent employés dans le cadre de cette étude, afin dévaluer les liens entre certaines caractéristiques personnelles et environnementales et la résilience. Un questionnaire sociodémographique a aussi permis d’obtenir un profil sociodémographique des adolescents. Afin de déterminer le statut des participants, une question sur l'appartenance culturelle a été incluse. Les participants autochtones devaient préciser leur lieu de résidence et celui de leurs parents, de même qu'ils devaient indiquer leur langue maternelle. Enfin, une question visant à évaluer spécifiquement la présence d'agression à caractère sexuel dans l'histoire des participants a été incluse (As-tu eu une relation sexuelle ou subi un geste sexuel ou été forcé à faire un geste sexuel à quelqu'un alors que tu ne voulais pas?)

Une partie du questionnaire « Inventaire de problèmes familiaux » (Thériault, Cyr, \& Wright, 1996) a été utilisée afin d'identifier des évènements de vie vécus dans l'enfance et l'adolescence. Il présente 12 évènements où l'adolescent doit indiquer s'il a vécu ou non la situation (p.ex. : violence familiale, séparation, abandon des enfants par un des parents, alcoolisme dans la famille).

Le Trauma Symptom Checklist for Children (TSC-C, Brière, 1989), dans sa version française (Wright \& Sabourin, 1996) a permis d'évaluer la symptomatologie psychologique. Il comprend six échelles portant respectivement sur l'anxiété, la dépression, le stress post-traumatique, les préoccupations sexuelles, la dissociation et la colère. Tel qu'employé dans certaines recherches (Elklit, Due, \& Christiansen, 2009), le score total obtenu a été utilisé pour évaluer le niveau de détresse psychologique. Ainsi, plus un adolescent affiche un score élevé, plus il présente de symptômes psychologiques et un niveau de détresse élevé et moins il est considéré résilient. Les jeunes âgés de plus de 8 ans répondent aux 54 items à l'aide d'une échelle de fréquence en quatre points (jamais, parfois, souvent, presque toujours). Deux échelles de validité sont incluses afin d'évaluer si l'enfant dénie ou intensifie les symptômes. La structure factorielle, sa validité de convergence et la cohérence interne de la traduction ont été démontrées à la suite d'une étude réalisée auprès de 1556 enfants (Jouvin et al., 2001). 
Léchelle d'estime de soi (EES - Rosenberg, 1955, traduction canadienne-française de Vallières \& Vallerand, 1990) est un bref questionnaire auto-administré constitué de 10 affirmations couvrant différents aspects de l'estime de soi (p. ex. : Je considère que j’ai au moins autant de valeur que les autres). L'EES comprend une échelle de type Likert en 4 points (de $1=$ tout à fait en désaccord à 4 = tout à fait d'accord). Cette échelle possède de bonnes qualités psychométriques comparables à la version originale anglaise. La consistance interne varie de 0,70 à 0,90 et a été évaluée à partir de quatre études portant sur des échantillons d'étudiants de niveau collégial (Vallières \& Vallerand, 1990). La fidélité test-retest a quant à elle été jugée équivalente à la version originale $(r=0.84)$.

Linventaire des attitudes parentales (Child's Attitude Toward the Mother/Father; Giuli \& Hudson, 1977, version francophone abrégée; Pauzé, Toupin, \& Déry, 1993) a été utilisé afin dévaluer la perception qu'a l'adolescent de la qualité des relations qu'il entretient avec chacun de ses parents (ou beaux-parents le cas échéant). Le jeune évalue cette relation en répondant aux 10 items sur une échelle en 5 points (de $1=$ rarement ou jamais à $5=$ souvent ou tout le temps). Les cohérences internes des versions abrégées de notre échantillon sont adéquates (alpha de cronbach $=0,92$ et 0,96 pour les versions mère et père respectivement), et comparables à celles de la version longue anglophone (Hudson, 1992).

La traduction française (Daigneault, 2008) de la sous-échelle « relation avec les autres » du Child and Youth Resilience Measure (CYRM, Ungar \& Liebenberg, 2005) a été utilisée pour évaluer le soutien reçu par les pairs (l'alpha de Cronbach de notre échantillon est de 0,55). Il est à noter que l'alpha de la version originale de cette échelle est de 0,76. Cette sous-échelle comprend trois items évalués sur une échelle en cinq points où les participants indiquaient à quel point chaque énoncé leur ressemblait (de $1=$ pas du tout à 5 = énormément). Deux items supplémentaires ont permis d'évaluer la capacité de l'adolescent à aller chercher de l'aide dans sa communauté, l'un provenant du CYRM (Sais-tu où aller pour obtenir de l'aide dans ta communauté?) et l'autre étant un item composite permettant de voir si l'adolescent a eu recours à l'aide d'un professionnel à l'école (ex. : psychologue, travailleur social, psychoéducateur).

\section{Stratégies d'analyses}

Dans un premier temps, les données ont fait l’objet d’analyses préliminaires afin de vérifier la normalité des données. Des chi-carrés ont ensuite été effectués afin d'évaluer les différences observées concernant les événements de vie entre les deux populations (adolescents autochtones vs caucasiens). Par la suite, des analyses corrélationnelles ont été effectuées dans le but d'évaluer la présence de relations significatives entre les données sociodémographiques, les événements vécus dans l'enfance, l'estime de soi, l'aide et le soutien communautaire et la qualité des relations avec les parents et la résilience psychologique. En outre, ces analyses ont permis de s'assurer de l'absence de problèmes de multicolinéarité avant l'analyse de régression. Une analyse de régression multiple de type séquentiel a donc ensuite été réalisée. Pour cette analyse, toutes les variables indépendantes à létude ont été incluses dans le modèle, afin de mieux comprendre leur influence respective sur la résilience.

\section{Résultats}

Les résultats des chi-carrés concernant les événements de vie apparaissent au tableau 1 et montrent qu'il y a davantage de précarité financière, d'alcoolisme, de violence conjugale, de violence physique envers les enfants, d'incarcération d'un membre de la famille, et d'abandon d'enfants 
First Peoples Child \& Family Review, Volume 7, Number 1, 2012

Tableau 1. Différences concernant les événements de vie entre les adolescents autochtones et caucasiens

\begin{tabular}{|c|c|c|c|c|}
\hline & $\begin{array}{l}\text { Autochtones } \\
\qquad \begin{array}{c}(\mathrm{n}=61) \\
(\%)\end{array}\end{array}$ & $\begin{array}{c}\text { Caucasiens } \\
(\mathrm{n}=166) \\
(\%)\end{array}$ & $\begin{array}{c}\text { Total } \\
(\mathrm{n}=227) \\
(\%)\end{array}$ & $x^{2}$ \\
\hline Problèmes d'argent & 45,0 & 28,5 & 32,9 & $5,44^{*}$ \\
\hline Divorce & 44,8 & 39,9 & 41,2 & 0,43 \\
\hline Violence conjugale (physique) & 23,3 & 5,5 & 10,3 & $15,04^{* * *}$ \\
\hline Violence conjugale (verbale) & 80,0 & 59,3 & 25,4 & $9,79^{* *}$ \\
\hline Violence physique envers enfants & 11,7 & 3,7 & 5,8 & $5,15^{\star}$ \\
\hline Violence verbale envers enfants & 18,3 & 16,6 & 17,0 & 0,10 \\
\hline Incarcération d'un membre de la famille & 20,3 & 6,7 & 10,3 & $8,82^{* *}$ \\
\hline Alcoolisme dans la famille & 45,8 & 21,2 & 27,7 & $13,09^{* * *}$ \\
\hline Abandon d'enfants par les parents & 15,3 & 3,0 & 6,3 & $11,08^{\star * *}$ \\
\hline Recours à l'aide d'un professionnel & 41,0 & 48,8 & 46,7 & 1,09 \\
\hline Avoir été agressé(e) sexuellement & 11,5 & 13,9 & 13,2 & 0,22 \\
\hline
\end{tabular}

${ }^{\star} \mathrm{p}<0,05 ;{ }^{* *} \mathrm{p}<0,01 ;{ }^{* *} \mathrm{p}<0,001$.

chez les adolescents autochtones. Les résultats sont comparables pour les deux populations en ce qui concerne le divorce, le recours à l'aide d'un professionnel et l'agression sexuelle. En somme, les résultats confirment notre hypothèse voulant que les adolescents autochtones aient vécu plus d'évènements de vie en général, mais non en ce qui concerne plus spécifiquement l'agression sexuelle où les taux sont similaires.

Afin d'évaluer la présence de relations significatives entre les différentes variables à létude, des corrélations se sont révélées significatives pour les trois hypothèses de recherche avancées en regard de la résilience considérée sous l'angle du faible niveau de détresse psychologique (TSC-C). Ainsi, l'estime de soi semble liée négativement à la détresse psychologique, donc plus l'estime de soi des adolescents est élevée, moins il y a de détresse psychologique. Les résultats suggèrent également que la qualité des relations avec les parents est associée à une meilleure santé psychologique. Ainsi, des relations adéquates avec la mère ou le père sont associées positivement à l'absence de détresse psychologique. Enfin, la capacité de l'adolescent à rechercher de l'aide dans sa communauté, de même que le soutien des amis présentent une relation négative avec la détresse psychologique. Toutefois, le fait de recevoir de l'aide professionnelle est associé à davantage de détresse psychologique chez les adolescents. Par ailleurs, être autochtone est relié à un niveau plus faible d’estime de soi et à plus d'évènements de vie.

Les résultats de l'analyse de régression (voir tableau 3 ) indique que six principaux facteurs sont significatifs et expliquent $34 \%$ (R2 ajusté) de la variance observée, à savoir, en ordre d'importance, l'estime de soi $(\beta=-0,29)$, avoir vécu une agression sexuelle $(\beta=-0,23)$, le nombre dévénements de vie négatifs $(\beta=0,15)$, l'aide communautaire $(\beta=-0,13)$, être d'origine autochtone $(\beta=-0,13)$ et enfin le sexe de l'adolescent $(\beta=0,13)$. Les résultats indiquent donc que l'estime de soi et la capacité à aller chercher de l'aide dans la communauté sont les facteurs de protection les plus susceptibles de contribuer à la résilience lorsque les autres variables sont contrôlées. Par ailleurs, les résultats suggèrent que le fait deêtre de sexe féminin, d'avoir subi une agression sexuelle et 


\section{Factors de Résilience chez des adolescents}

Tableau 2. Corrélations des variables à l'étude

\begin{tabular}{|c|c|c|c|c|c|c|c|c|c|c|}
\hline Variables & 1 & 2 & 3 & 4 & 5 & 6 & 7 & 8 & 9 & 10 \\
\hline 1. TSC-C & - & & & & & & & & & \\
\hline 2. Estime de soi & $-.41^{\star * *}$ & - & & & & & & & & \\
\hline 3. Soutien mère & $-.24^{\star * *}$ & $.24^{* * *}$ & - & & & & & & & \\
\hline 4. Soutien père & $-.26^{* * *}$ & $.23^{* *}$ & $.42^{* * *}$ & - & & & & & & \\
\hline 5. Soutien pairs & $-.14^{*}$ & $.22^{* *}$ & $.29^{* * *}$ & $-.31^{* * *}$ & _- & & & & & \\
\hline 6. Aide professionnelle & $.26^{* * *}$ & $-.16^{*}$ & -.08 & $-.13^{*}$ & -.04 & - & & & & \\
\hline 7. Aide communautaire & $-.22^{\star *}$ & $.18^{* *}$ & $.17^{* *}$ & $.17^{*}$ & $.19^{* *}$ & .04 & - & & & \\
\hline 8. Évènements de vie & $.33^{* * *}$ & $-.23^{* * *}$ & $-.24^{* * *}$ & $-.35^{* * *}$ & $-.14^{*}$ & $.18^{* *}$ & $-.15^{*}$ & - & & \\
\hline 9. Agression sexuelle & $.35^{\star * *}$ & -.05 & .00 & -.12 & -.01 & $.23^{* *}$ & -.06 & $.22^{* *}$ & - & \\
\hline 10. Sexe & $.17^{*}$ & -.03 & $.16^{*}$ & -.02 & $.28^{* * *}$ & $.18^{* *}$ & $.13^{*}$ & .05 & $.18^{* *}$ & - \\
\hline 11. Origine autochtone & -.03 & $-.15^{\star}$ & -.09 & -.09 & .03 & .07 & -.05 & $.22^{* *}$ & -.04 & -.02 \\
\hline
\end{tabular}

${ }^{\star} \mathrm{p}<0,05 ;{ }^{* *} \mathrm{p}<0,01 ;{ }^{* * *} \mathrm{p}<0,001$.

Tableau 3. Analyses de régression multiple de type séquentiel des facteurs exerçant une influence sur la détresse psychologique (résilience)

\section{B}

$\operatorname{ET}(\mathrm{B}) \quad \operatorname{Beta}(\beta)$ $\mathrm{t}$ Sig

\begin{tabular}{|c|c|c|c|c|c|}
\hline \multicolumn{6}{|l|}{ Étape 1} \\
\hline Sexe & 3,92 & 2,20 & 0,11 & 1,78 & 0,08 \\
\hline Origine autochtone & $-3,36$ & 2,53 & $-0,08$ & $-1,33$ & 0,19 \\
\hline Nb. événements de vie & 5,67 & 1,25 & 0,29 & $4,53^{\star * *}$ & 0,00 \\
\hline Agression sexuelle & 14,25 & 3,42 & 0,26 & $4,17^{\star \star \star}$ & 0,00 \\
\hline \multicolumn{6}{|l|}{ Étape 2} \\
\hline Sexe & 4,64 & 2,15 & 0,13 & $2,16^{*}$ & 0,03 \\
\hline Origine autochtone & $-5,51$ & 2,34 & $-0,13$ & $-2,38^{*}$ & 0,02 \\
\hline Nb. événements de vie & 3,01 & 1,22 & 0,15 & $2,47^{\star}$ & 0,01 \\
\hline Agression sexuelle & 12,46 & 3,16 & 0,23 & $3,94^{\star * *}$ & 0,00 \\
\hline Estime de soi & $-1,01$ & 0,21 & $-0,29$ & $-4,94^{\star * *}$ & 0,00 \\
\hline Soutien mère & $-0,10$ & 0,05 & $-0,11$ & $-1,79$ & 0,07 \\
\hline Soutien père & $-0,03$ & 0,05 & $-0,04$ & $-0,61$ & 0,54 \\
\hline Soutien pairs & $-0,05$ & 0,53 & $-0,01$ & $-0,10$ & 0,92 \\
\hline Aide professionnelle & 3,97 & 2,11 & 0,11 & 1,89 & 0,06 \\
\hline Aide communautaire & $-1,97$ & 0,90 & $-0,13$ & $-2,20^{*}$ & 0,03 \\
\hline
\end{tabular}

$\mathrm{R} 2$ ajusté $=0,34^{* * *} ;{ }^{*} \mathrm{p}<.05 ;^{* *} \mathrm{p}<.01 ;^{* * *} \mathrm{p}<.000$ 
First Peoples Child \& Family Review, Volume 7, Number 1, 2012

d’avoir été exposé à plusieurs événements stressants constituent des facteurs de risques reliés à une moins grande résilience. Enfin, le statut autochtone s'avère négativement corrélé avec le niveau de détresse lorsque les autres variables de l'étude sont contrôlées, alors que le soutien reçu des parents et des pairs, de même que l'aide professionnelle ne sont plus liés à la détresse psychologique rapportée.

\section{Discussion}

Lobjectif principal de cette recherche portait sur létude de facteurs de protection susceptibles d'exercer une influence positive sur la résilience psychologique. Ainsi, tel qu'attendu, les résultats des analyses corrélationnelles se sont avérés significatifs pour quatre des cinq hypothèses avancées. Une analyse de régression séquentielle a permis de retenir l'estime de soi comme facteur le plus significatif en regard de la résilience. Certaines variables furent aussi considérées en tant que facteurs de risque potentiels.

\section{Évènements de vie et agression sexuelle}

Les résultats obtenus révèlent que les adolescents autochtones de notre échantillon sont exposés à davantage de problèmes psychosociaux que les adolescents caucasiens, ce qui correspond aux constats avancés dans d'autres recherches (Blackstock \& Trocmé, 2004; Brownridge, 2003; Kirmayer, Brass, \& Tait, 2000). Toutefois, les résultats indiquent qu'il n'y a pas de différence significative en regard de la prévalence de l'agression sexuelle entre les deux populations, ce qui semble aller à l'encontre de résultats récemment présentés par Collin-Vézina et ses collègues (2009). Cependant, on peut penser que la taille réduite de l'échantillon autochtone dans la présente étude limite la portée des résultats. Outre les facteurs de sous-dévoilement communs aux personnes agressées sexuellement, certains aspects pouvant s'avérer plus délicats en contexte autochtone pour des raisons historiques (p. ex. : méfiance envers le système de justice), socioculturelles (p. ex. : famille élargie) et parfois géographiques (p. ex. : isolement et manque de ressources) pourraient expliquer les résultats. Ainsi, la culpabilité ressentie ou la peur d'exposer un proche à la justice, la crainte de mettre en péril l'unité familiale, et enfin les représailles éventuelles par des gens de la communauté sont toujours possibles (Native Women's Association of Canada [NCWA], 1994; Picard, 2004). D’autre part, on peut aussi émettre l'hypothèse que la situation géographique de la communauté autochtone d’où provenaient les participants, notamment la proximité de la réserve avec d'autres agglomérations urbaines, a pu exercer une influence positive : permettre dans certains cas, contrairement aux réserves éloignées, une accessibilité accrue à l'obtention de services d'aide et de conseils pour les familles en difficulté. Ajoutons que notre échantillon autochtone fréquentait un établissement scolaire, un lieu qui peut s'avérer un agent de prévention et de sensibilisation quant à la problématique liée à l'agression sexuelle. Enfin, il est aussi possible que l'agression sexuelle soit moins présente chez les personnes autochtones plus jeunes que chez les plus âgés, qui pour leur part furent exposées au traumatisme du régime des pensionnats. Néanmoins, il importe que d'autres études soient réalisées auprès déchantillons plus vastes et dans diverses communautés, afin d’obtenir un meilleur estimé de la prévalence de l'agression sexuelle en milieu autochtone.

\section{Facteurs de risque et résilience}

Les résultats de la régression suggèrent que plusieurs facteurs affectent la résilience. À ce titre, les résultats obtenus semblent démontrer que les adolescentes de léchantillon seraient moins 
résilientes, ce qui pourrait révéler un impact en regard du sexe. Ainsi, les résultats sont similaires à plusieurs recherches précédentes indiquant que les filles présentent davantage de détresse psychologique que les garçons (Ayotte, Fournier, \& Riberdy, 2009; Hong, 1995; Picard, Claes, Melançon, \& Miranda, 2007), mais contraires à ceux obtenus par Lafromboise et al. (2006) auprès d'adolescents plus jeunes et où le sexe nétait pas relié à la résilience. Il faut toutefois considérer ici la définition de la résilience restreinte aux seuls symptômes psychologiques, ce qui peut conduire à des inférences erronées. De plus, il est possible que notre mesure de symptômes n'ait pas pu détecter ces différences, notamment parce que les garçons présentent plus souvent des problèmes externalisés qu'internalisés (p. ex. : Leadbeater, Kuperminc, Blatt, \& Hertzog, 1999). Il est également possible que les garçons avouent plus difficilement leur détresse psychologique que les filles (Dumont, 2000), ce qui expliquerait pourquoi ils semblent plus résilients dans notre échantillon. En outre, les exigences sociales et émotionnelles survenant durant l'adolescence peuvent être particulièrement éprouvantes pour les filles autochtones, qui se retrouvent alors plus souvent dans des situations à risque, pouvant ainsi compromettre leur confiance en leur capacité à surmonter les difficultés (Lafromboise et al., 2006).

Le fait d’avoir vécu plusieurs évènements stressants constitue un risque dêtre moins résilient. En effet, ces résultats confirment ceux de DuMont et ses collègues (2007) indiquant que les gens plus résilients ont été moins fréquemment en contact avec des situations stressantes que ceux affichant un niveau de résilience moindre. De plus, les résultats des analyses révèlent que l'agression sexuelle est un évènement de vie qui exerce une influence plus importante que le nombre d'évènements de vie difficiles. L'impact d'une agression sexuelle sur le fonctionnement d'un individu pourrait donc être plus néfaste que d'autres types d'évènements de vie. D’ailleurs, les résultats d'une étude longitudinale récente indiquent qu'au cours de l'enfance, l'agression sexuelle a un effet plus délétère que l'agression physique sur la santé psychologique à lâge adulte (Fergusson, Boden, \& Horwood, 2008).

La corrélation entre le statut autochtone et la résilience s’est avérée non significative. Toutefois, les résultats de l'analyse de régression montrent que les adolescents autochtones présentent davantage de résilience que les adolescents non-autochtones. Les jeunes autochtones de l'échantillon ont été davantage exposés aux événements de vie délétères que leurs homologues caucasiens, en plus de présenter une estime de soi un peu plus faible, ce qui pourrait expliquer pourquoi la relation entre le statut autochtone et la résilience ne ressort pas lorsque le nombre d'évènements de vie et l'estime de soi ne sont pas contrôlés. Ainsi, lorsque les variables indépendantes de notre étude sont contrôlées dans l'analyse de régression, l'effet du statut autochtone ressort (ce qu'on appelle un effet de suppression, Mackinnon, Krull, \& Lockwood, 2000). D’autres études seront nécessaires afin de mieux comprendre ce qui fait que les adolescents autochtones s'avèrent un peu plus résilients que leurs homologues caucasiens. On peut penser que certains facteurs culturels (non mesurés) propres aux personnes autochtones pourraient avoir exercé une influence positive sur la résilience : par exemple le sentiment d'appartenance culturelle (Lafromboise et al., 2006), la spiritualité, ou la pratique de rites de guérison, des dimensions liées à la notion de cercle sacré (Chansonneuve, 2005), un symbole influant et reconnu au sein des cultures autochtones (Muckle \& Dion, 2008).

\section{Facteurs de protection et résilience}

Les corrélations concernant les liens possibles entre l'estime de soi et le soutien parental et social en regard de la résilience se sont révélées statistiquement significatives, tel qu’avancé par les 
études précédentes sur la résilience (Libby et al., 2008; Scott Heller et al., 1999). Ces facteurs ont ensuite été intégrés dans un modèle de régression qui révèle que l'estime de soi est le facteur qui exerce l'influence la plus significative sur la résilience chez les adolescents de létude. Ces résultats sont conformes à ceux d'une autre étude proposant que l'estime de soi favorise la résilience en exerçant un effet modérateur sur le stress généré par des événements de vie délétères (Neighbors, Forehand, \& McVicar, 1993). De plus, une meilleure estime de soi favoriserait l'emploi de meilleures stratégies d'ajustement tout en augmentant la confiance en soi, améliorant ainsi les capacités à réagir face à l’adversité (Dumont \& Provost, 1999; Thoits, 1995).

Les résultats obtenus semblent aussi démontrer que la perception de la qualité des relations parentales n'est pas reliée à la résilience lorsqu'on prend en compte d'autres facteurs, bien que la qualité de la relation avec la mère soit marginalement significative. Pourtant, Goldstein et Brooks (2005) expliquent qu'un style parental soutenant fait de réciprocité, de chaleur et de compréhension serait un facteur de résilience en favorisant un sentiment de compétence personnelle et sociale. Ces mêmes auteurs ajoutent que cela pourrait avoir un impact sur les facteurs de risques affectant la santé mentale. Les résultats obtenus pourraient s'expliquer par le fait que les adolescents se situent dans une étape développementale souvent caractérisée par un certain détachement envers leurs parents, ce qui peut être lié à des aspects à la fois identitaires, mais relevant aussi de l'exploration de nouveaux rôles sociaux (Cloutier \& Drapeau, 2008). Dans le cadre de la présente étude, une mesure de la qualité de la relation a été utilisée plutôt que d'autres types de mesure, tel le soutien parental, ce qui a également pu influencer les résultats. Il serait donc intéressant d'utiliser plus d'une mesure de la relation parent-enfant (qualité, soutien, attachement, etc.) afin de mieux comprendre l'influence de cette variable sur la résilience.

La capacité à rechercher le soutien disponible dans la communauté peut également contribuer à la résilience. Ainsi, comme avancé dans d'autres recherches, cette variable serait associée à un meilleur ajustement psychologique et contribuerait à la résilience (Armstrong et al., 2005; Horton \& Wallander, 2001). À cet effet, la disponibilité des ressources, de même que la manière dont les adolescents emploient celles-ci sont d'ailleurs des variables susceptibles de favoriser un développement sain par opposition à l'apparition de problèmes psychologiques (Compas, 1987). Ces dernières variables nont pas été étudiées dans le cadre de la présente étude. En effet, c'est la capacité de l'adolescent à chercher du soutien qui a été évaluée, ce qui ne permet pas de savoir si des ressources sont disponibles, ou encore comment elles sont utilisées. Par ailleurs, la présente étude a mesuré l'aspect communautaire avec l'aide d'une seule question. Cette dimension gagnerait donc à être évaluée de manière plus élaborée, d’autant plus quelle revêt de l'importance pour les peuples autochtones (Muckle \& Dion, 2008), par exemple en évaluant la participation culturelle, l'identité culturelle, ou encore la discrimination raciale. Enfin, seulement quelques énoncés de la mesure de résilience (CYRM) ont été utilisés dans le cadre de la présente étude pour évaluer le soutien social et communautaire. Il serait important que des études futures l'utilisent dans sa version complète lorsque la validation française sera terminée et que nous aurons plus de données quant à sa validité discriminante et prédictive en regard des aspects culturels de la résilience.

Alors que les résultats des corrélations montraient une association entre le soutien par les pairs et la résilience, l'analyse de régression révèle que cette variable n'est plus significative. Ainsi, l'estime de soi et la capacité à rechercher du soutien dans sa communauté semblent des facteurs de protection ayant plus d'impact sur la résilience. Étant donné que les relations avec les pairs représentent une dimension importante à l'adolescence, il serait important d'en évaluer l'influence sur la résilience à l'aide de mesures plus variées. En effet, il est plausible que l'échelle employée 
dans le cadre de la présente étude n’ait pu permettre d'évaluer de façon adéquate le soutien reçu par les pairs en raison du fait qu'elle était trop restreinte et ne couvrait qu'un seul aspect.

Contrairement à ce qui était attendu, les résultats concernant l'aide professionnelle reçue à l'école se sont révélés associés à davantage de détresse psychologique. Cela semble indiquer que l'aide professionnelle reçue ne pourrait être conceptualisée en tant que facteur de résilience dans la présente étude. Néanmoins, ces résultats sont cohérents dans la mesure où les gens consultent habituellement un professionnel lorsqu'ils éprouvent des symptômes. Ces résultats peuvent cependant être le fait du schème transversal employé qui ne considère qu'un temps de mesure. À cet effet, les résultats indiquent néanmoins que les adolescents éprouvant des difficultés psychosociales ont recours à l'aide des professionnels. Ainsi, il serait intéressant d'évaluer à plus long terme si les adolescents qui ont reçu de l'aide présentent, après que l'intervention soit terminée, moins de symptômes, ce qui pourrait alors signifier que l'aide reçue a été bénéfique et susceptible de contribuer à la résilience.

\section{Limites de l’étude}

Les limites de la présente étude ont d'une part trait à l'opérationnalisation de la résilience qui n'a pu permettre d'en saisir toute la complexité puisqu'elle ne considère que l'aspect symptomatologique. De plus, la nature transversale ainsi que le devis corrélationnel ne nous permettent au mieux que des inférences quant à l'influence exercée par chacune des variables en regard de la résilience. Enfin, le nombre d'adolescents autochtones et la faible proportion de victimes d'agression sexuelle de notre échantillon limitent la portée des résultats. En dépit de ces limites, cette étude présente un intérêt certain, puisquelle est une des rares à soumettre des résultats de type quantitatifs à partir d'un échantillon d'adolescents autochtones.

\section{Conclusion}

Les résultats de la présente étude correspondent aux connaissances actuelles avancées dans la recherche sur la résilience et suggèrent l'importance d'utiliser une approche écosystémique qui implique non seulement les facteurs individuels, mais aussi les transactions avec l'environnement. En effet, les résultats obtenus semblent congruents avec la recherche actuelle puisque l'estime de soi et le soutien communautaire se sont avérés significatifs. En effet, tel qu'avancé par d'autres chercheurs, ces facteurs sont associés au bien-être psychologique, favorisent les interactions sociales, contribuent à une plus grande sécurité émotionnelle et protègent la personne des effets délétères et de leur expression sur le plan de la santé mentale (Armstrong et al., 2005; Chen Yi-Feng et al., 2008; Rosenberg et al., 1995). À la lumière des analyses obtenues, on peut donc penser que l'estime de soi est un important facteur de protection pour une trajectoire résiliente. Ainsi, des interventions en amont visant à améliorer l'estime de soi, à promouvoir la qualité des relations avec les parents et l'initiative des adolescents à utiliser le soutien disponible dans leur environnement seraient susceptibles de contribuer à la résilience en diminuant la détresse psychologique. Néanmoins, les résultats obtenus doivent aussi être nuancés, les trajectoires résilientes pouvant être multiples en raison du fait qu'il s'agit d'un processus dynamique et complexe (Anaut, 2002; Lemay, 2001; Luthar et al., 2000).

Les résultats présentés nous incitent aussi à souligner l'importance d'investir davantage dans la recherche afin de poursuivre les efforts de compréhension du processus résilient et des facteurs susceptibles d'en favoriser l'accroissement, notamment chez les victimes d'agression sexuelle 
First Peoples Child \& Family Review, Volume 7, Number 1, 2012

en milieu autochtone. En effet, la violence sexuelle chez les peuples autochtones est complexe, et devrait faire l'objet d'une approche sensible aux dimensions à la fois historiques, sociales, politiques et culturelles (Muckle \& Dion, 2008). Ainsi en contexte multiculturel, et en particulier chez les personnes autochtones, une approche clinique des traumatismes, des évènements de vie et de leurs impacts, qui soriente habituellement sur le fonctionnement individuel, gagnerait à intégrer les systèmes communautaires en adoptant une perspective de la résilience qui soit écologique (Blackstock \& Trocmé, 2004; McCubbin, McCubbin, Thompson, \& Thompson, 1998; Tummala-Narra, 2007).

\section{Remerciements}

Les auteurs souhaitent remercier (Tshinashkumitin) tous les adolescents et adolescentes qui ont bien voulu participer à cette étude en acceptant de partager plusieurs aspects de leur vie personnelle. Merci également à monsieur Bouchard, directeur enthousiaste, qui nous a ouvert les portes de son établissement d'enseignement, de même que les enseignants de cette école. Nous remercions les assistantes de recherche, notamment Marie-Frédéric Bouchard et Delphine Lagacé, de même que les intervenants autochtones pour leur précieuse collaboration à ce projet. Cette étude a été réalisée dans le cadre des recherches du Centre de recherche interdisciplinaire sur les problèmes conjugaux et les agressions sexuelles (CRIPCAS). Elle a été réalisée avec le soutien financier de l'Université du Québec à Chicoutimi, par le biais du programme d'aide institutionnel à la recherche, octroyé à la 2ème auteure.

\section{Références}

Anaut, M. (2002). Trauma, vulnérabilité et résilience en protection de l’enfance. Connexions, 1, 101-118.

Armstrong, M. I., Birnie-Lefcovitch, S., \& Ungar, M. T. (2005). Pathways between social support, family well being, quality of parenting and child resilience : What we know. Journal of Child and Family Studies, 14, 269-281.

Ayotte, V., Fournier, M., \& Riberdy, H. (2009). La détresse psychologique des enfants et des adolescents montréalais... l'expression de différentes réalités? Montréal : Direction de santé publique de l’agence de la santé et des services sociaux de Montréal.

Barker-Collo, S. L. (1999). Reported symptomatoly of native Canadian and Caucasian females sexually abused in childhood: A comparison. Journal of Interpersonal Violence, 14, 747-759.

Blackstock, C., \& Trocmé, N. (2004). Community based child welfare for aboriginal children: Supporting resilience through structural change. Toronto: First Nations Child and Family Caring Society et Centre of excellence for Child Welfare.

Brave Heart, M. Y. H., \& DeBruyn, L. M. (1998). The American Indian holocaust: Healing historical unresolved grief. The Journal of the National Center, 8, 60-82.

Brière, J. (1989). Trauma symptom checklist for children (TSC-C). Los Angeles, CA: University of Southern California Press.

Brownridge, D. A. (2003). Male partner violence against Aboriginal women in Canada: An empirical analysis. Journal of Interpersonal Violence, 18, 65-83.

Carlton, B. S., Goebert, D. A., Miyamoto, R. H., Andrade, N. N., Hishinuna, E. S., Makini, G. K., et al. (2006). Resilience, family adversity and well-being among Hawaiian and non-Hawaiin adolescents. International Journal of Social Psychiatry, 52, 291-308.

Centre des Premières Nations et Organisation nationale de la santé autochtone. (2006). Enquête régionale longitudinale sur la santé des Premières Nations (ERS) 2002 - 2003. Le rapport pour les peuples. Ottawa. 


\section{Factors de Résilience chez des adolescents}

Centre national d'information sur la violence dans la famille. (2006). Abus sexuel à l'égard des enfants. Récupéré le 5 septembre 2008 de http://www.phac-aspc.gc.ca/nc-cn/.

Chansonneuve, D. (2005). Retisser nos liens : Comprendre les traumatismes vécus dans les pensionnats indiens par les Autochtones. Ottawa : Fondation autochtone de guérison.

Chen Yi-Feng, N., Huang, X., \& Tjosvold, D. (2008). Similarity in gender and self-esteem for supportive peer relationships: The mediating role of cooperative goals. Journal of Applied Social Psychology, 38, 1147-1178.

Cloutier, R., \& Drapeau, S. (2008). Psychologie de l’adolescence, 3e édition. Québec : Gaëtan Morin éditeur.

Collin-Vézina, D., Dion, J., \& Trocmé, N. (2009). Sexual abuse in Canadian Aboriginal communities: A comprehensive review of conflicting evidence. Pimatisiwin: A Journal of Aboriginal and Indigenous Community Health, 7, 27-47.

Commission Royale sur les Peuples Autochtones, Dussault, R., Erasmus, G., Chartrand, P. L. A. H., Meekison, J. P., Robinson, V., Sillett, M. et al. (1996). Rapport de la commission royale d'enquête sur les peuples autochtones. Affaires indiennes et du Nord Canada.

Compas, B. E. (1987). Coping with stress during childhood and adolescence. Psychological Bulletin, 100, 393-403.

Constantine, A. N., Benard, B., \& Diaz, M. (1999). Measuring protective factors and resilience traits in youth: The healthy kids resilience assessment. Paper presented at the Seventh annual meeting of the society for prevention research.

Corrado, R. R., \& Cohen, I. M. (2003). Profils de la santé mentale d’un échantillon d'Autochtones de la ColombieBritannique survivants du régime canadien des pensionnats. Ottawa : Fondation autochtone de guérison.

Daigneault, I. (2008). Traduction française du Child and Youth Resilience Measure (CYRM). Université de Montréal.

Daigneault, I., Cyr, M., \& Tourigny, M. (2007). Exploratory of recovery trajectories and associated factors in sexually abused adolescents. Journal of Aggression, Maltreatment and Trauma, 14, 164-184.

Daigneault, I., Hébert, M., \& Tourigny, M. (2007). Personal and interpersonal characteristics related to resilient developmental pathways of sexually abused adolescents. Child and Adolescents Psychiatric Clinics of North America, 16, 415-434.

Drapeau, S., Saint-Jacques, M.-C., Lépine, R., Bégin, G., \& Bernard, M. (2007). Processes that contribute to resilience among youth in foster care. Journal of Adolescence, 30, 977-999.

Dumont, M., \& Provost, M. A. (1999). Resilience in adolescents: Protective role of social support, coping strategies, self-esteem, and social activities on experience of stress and depression. Journal of Youth and Adolescence, 28, 343-363.

DuMont, K. A., Widom, S. C., \& Czaja, J. S. (2007). Predictors of resilience in abused and neglected children grown-up: The role of individual and neighborhood characteristics. Child Abuse \& Neglect, 31, 255-272.

Dumont, M. (2000). Expérience du stress à l’adolescence. Journal International de Psychologie, 35, 194-206.

Elklit, A., Due, L., \& Christiansen, D. M. (2009). Predictors of acute stress symptoms in rape victims. Traumatology, $15,38-45$.

Fergusson, D. M., Boden, J. M., \& Horwood, J. L. (2008). Exposure to childhood sexual and physical abuse and adjustment in early adulthood. Child Abuse and Neglect, 32, 607-619.

Finkelhor, D. (1990). Early and long-term effects of child sexual abuse: An update. Professional Psychology: Research and Practice, 21, 325-330.

Fortin, B. (1989). Ce que vous devez savoir sur l'estime de soi. Association canadienne pour la santé mentale : Outaouais.

Giuli, C. A., \& Hudson, W. W. (1977). Assessing parent-child relationship disorders in clinical practice: The child's point of view. Journal of Social Service Research, 1, 77-92.

Goldstein, S., \& Brooks, R. B. (2005). Resilience in children. New York: Springer.

Golse, B. (2006). La résilience: Concept utile ou concept valide? Archives de pédiatrie, 13, 1354-1357.

Harter, S., Waters, P., \& Whitesell, N. R. (1998). Relational self-worth: Differences in perceived worth as a person across interpersonal contexts among adolescents. Child Development, 69, 756-766. 
First Peoples Child \& Family Review, Volume 7, Number 1, 2012

Harter, S., Whitesell, N. R., \& Junkin, L. J. (1998). Similarities and differences in domain-specific and global selfevaluations of learning-disabled, behaviorally disordered, and normally achieving adolescents. American Educational Research Journal, 35, 653-680.

Hong, Z.-R., McCarthy, P., \& Lawrenz, F. (2005). Psychosocial predictors of psychological distress in Taiwanese secondary school boys and girls. Sex Roles, 53(5/6), 419-431.

Horton, V., \& Wallander, J. L. (2001). Hope and social support as resilience factors against psychological distress of mothers who care for children with chronic physical conditions. Rehabilitation Psychology, 46, 382-399.

Hudson, W. W. (1992). Child's attitude toward father and mother scales. The WALMYR Assessment Scales Scoring Manual. Tempe, AZ: WALMYR.

Jendoubi, V. (2002). Estime de soi et éducation scolaire. République et canton de Genève : Service de la recherche en éducation.

Jiwani, Y., Gorkoff, K., Berman, H., Taylor, G., Vardy-Dell, G., \& Normandeau, S. (1999). Violence Prevention and the Girl Child: Phase One Report. Vancouver, BC: The Alliance of Five Research Centres on Violence.

Jouvin, E., Cyr, M., Thériault, C., \& Wright, J. (2001). Étude des qualités psychométriques de la traduction française du Trauma Symptom Checklist for Children (TSC-C). Document inédit, Université de Montréal.

Kegler, M., Oman, R., Vesely, S., McLeroy, K., Aspy, C., Rodine, S., et al. (2005). Relationships among youth assets and neighborhood and community resources. Health, Education and Behavior, 32, 380-397.

Kendall-Tackett, K. A., Williams, L. M., \& Finkelhor, D. (1993). Impact of sexual abuse on children: A review and synthesis of recent empirical studies. Psychological Bulletin, 113, 164-180.

Kirmayer, L. J., Brass, G. M., \& Tait, C. L. (2000). The mental health of aboriginal peoples: Transformations of identity and community. Canadian Journal of Psychiatry, 45, 607-616.

Kolar, K. (2011). Resilience: Revisiting the Concept and its Utility for Social Research. International Journal of Mental Health and Addiction, 9, 421-433.

LaFromboise, T. D., Hoyt, D. R., Oliver, L., \& Whitbeck, L. B. (2006). Family, community, and school influences on resilience among American Indian adolescents in the upper Midwest. Journal of Community Psychology, 34, 193-209.

Leadbeater, B. J., Kuperminc, G. P., Blatt, S. J., \& Hertzog, C. (1999). A multivariate model of gender differences in adolescents' internalizing and externalizing problems. Developmental Psychology, 35, 1268-1282.

LeClair, M., \& Associates. (1996). Profile of Aboriginal Sex Offenders. Ottawa, ON: Correctional Service Canada.

Lemay, M. (2001). La résilience devant la violence. Revue québécoise de psychologie, 22, 135-148.

Levan, M. B. (1989). Report on Child Sexual Abuse Needs Assessment. Paper presented at the Communities' Voice on Child Sexual Abuse. Yellowknife, NWT.

Libby, A. M., Orton, H. D., Beals, J., Buchwald, D., \& Manson, S. M. (2008). Childhood abuse and later parenting outcomes in two American Indian tribes. Child Abuse \& Neglect, 32, 195-211.

Luthar, S. S., Cicchetti, D., \& Becker, B. (2000). The construct of resilience: A critical evaluation and guidelines for future work. Child Development, 71, 543-562.

Lynskey, M. T., \& Fergusson, D. M. (1997). Factors protecting against the development of adjustment difficulties in young adults exposed to childhood sexual abuse. Child Abuse \& Neglect, 21, 1177-1190.

Mackinnon, D. P., Krull, J. L. \& Lockwood, C. M. (2000). Equivalence of the mediation, counfounding and suppression effect. Prevention Science, 1, 173.

MacDonald, G., \& O'Hara, K. (1996). Ten elements of mental health, its promotion and demotion: Implications for practice. Birmingham, United Kingdom: Society of Health Education \& Health Promotion Specialists.

MacMillan, H. L., Fleming, J. E., Streiner, D. L., Lin, E., Boyle, M. H., Jamieson, E., et al. (2001). Childhood abuse and lifetime psychopathology in a community sample. American Journal of Psychiatry, 158, 1878-1883. 


\section{Factors de Résilience chez des adolescents}

Maniglio, R. (2009). The impact of child sexual abuse on health: A systematic review of reviews. Clinical Psychology Review, 29, 647-657.

McClure, F. H., Chavez, D. V., Agars, M. D., Peacock, J. M., \& Matosian, A. (2008). Resilience in sexually abused women: Risk and protective factors. Journal of Family Violence, 23, 81-88.

McCubbin, H. I., McCubbin, M. A., Thompson, A. I., \& Thompson, E. A. (1998). Resiliency in ethnic families: A conceptual model for predicting family adjustment and adaptation. In H. I. McCubbin (Éd.), Resiliency in native American and immigrant families (pp. 1-46). CA: Thousand Oaks, Sage.

McEvoy, M., \& Daniluk, J. (1995). Wounds to the soul: The experiences of aboriginal women survivors of sexual abuse. Canadian Psychology, 36, 221-235.

McIvor, S. D., \& Nahanee, T. A. (1998). Aboriginal women: Invisible victims of violence. In K. Bonnycastler \& G. S. Rigakos (Éds.), Unsettling Truths: Battered Women, Policy, Politics, and Contemporary Research in Canada (pp. 63-70). Vancouver, BC: Collective Press.

Ministère de la Justice Canada. (2006a). Violence ou exploitation sexuelle des enfants et des adolescents: fiche d'information du ministère de la Justice du Canada. Récupéré le 29 novembre 2010, de http://canada.justice.gc.ca/en/ps/fm.

Ministère de la Justice Canada. (2006b). Violence ou exploitation sexuelle des enfants et des adolescents : fiche d’information du ministère de la Justice du Canada. Récupéré le 29 novembre 2010, de http://canada.justice.gc.ca/ en/ps/fm.

Muckle, F., \& Dion, J. (2008). Les facteurs de résilience et de guérison chez les autochtones victimes d'agression sexuelle. Revue québécoise de psychologie, 29, 1-14.

Mullen, P. E., Martin, J. L., Anderson, J. C., Romans, S. E., \& Herbison, G. P. (1996). The long-term impact of the physical, emotional, and sexual abuse of children: A community study. Child Abuse \& Neglect, 20, 7-21.

Neighbors, B., Forehand, R., \& McVicar, D. (1993). Resilient adolescents and interparental conflict. American Journal of Orthopsychiatry, 63, 462-471.

NWAC. (1994). Aboriginal women: Police charging policies and domestic violence. Winnipeg, MB: Native Women's Association of Canada.

Organisation nationale de la santé autochtone (ONSA). (2006). Enquête régionale longitudinale sur la santé des Premières Nations: Le rapport pour les peuples. Ottawa : Centre des Premières Nations.

Pauzé, R., Toupin, J., \& Déry, M. (1993). Inventaire des attitudes parentales. (Translation of the Index of Parental Attitudes). Unpublished manuscript, Université de Sherbrooke.

Pereda, N., Guilera, G., Forns, M., \& Gómez-Benito, J. (2009). The prevalence of child sexual abuse in community and student samples: A meta-analysis. Clinical Psychology Review, 29, 328-338.

Picard, P. (2004). L'intervention auprès des Premières Nations : la nécessité d'une approche intégrée. Québec: Groupe de recherche et d'interventions psychosociales en milieu autochtone (GRIPMA).

Picard, L., Claes, M., Melançon, C., \& Miranda, D. (2007). Qualité des liens affectifs parentaux perçus et détresse psychologique à l'adolescence. Enfance, 4, 371-392.

Pinkerton, J., \& Dolan, P. (2007). Family support, social capital, resilience and adolescent coping. Child and Family Social Work, 12, 219-228.

Putnam, F. W. (2003). Ten-year research update review: Child sexual abuse. Journal of American Academy of Child \& Adolescent Psychiatry, 42, 269-278.

Roos, P. E., \& Cohen, L. H. (1987). Sex roles and social support as moderators of life stress adjustment. Journal of Personality and Social Psychology, 52, 576-585.

Rosenberg, M., Schooler, C., Schoenbach, C., \& Rosenberg, F. (1995). Global self-esteem and specific self-esteem: Different concepts, different outcomes. American Sociological Review, 60, 141-156.

Scales, P. C., Benson, P., Leffert, N., \& Blyth. (2000). Contribution of developmental assets to the prediction of thriving among adolescents. Applied Developmental Science, 4, 27- 46. 


\section{First Peoples Child \& Family Review, Volume 7, Number 1, 2012}

Scott-Heller, S., Larrieu, J. A., D’Imperio, R., \& Boris, N. W. (1999). Research on resilience to child maltreatment: Empirical considerations. Child Abuse \& Neglect, 23, 321-338.

Seery, M. D., Holman, A. E., \& Cohen- Silver, R. (2010). Watever does not kill us: Cumulative lifetime adversity, vulnerability, and resilience. Journal of Personality and Social Psychology, 99, 1025-1041.

Stoltenborgh, M., Van IJzendoorn, M. H., Euser, E. M., \& Bakermans-Kranenburg, M. J. (2011). A global perspective on child abuse: Meta-analysis of prevalence around the world. Child Maltreatment, 26, 79-101.

Thériault, C., Cyr, M., \& Wright, J. (1996). Traduction et adaptation des problèmes familiaux vécus avant l'âge adulte de Courtois (1988). Document inédit : Université de Montréal.

Thoits, P. A. (1995). Stress, coping and social support processes: What are we? What next? Journal of Health and Social Behavior, 35, 53-79.

Tourigny, M., Gagné, M.-H., Joly, J., \& Chartrand, M.-E. (2006). Prévalence et cooccurrence de la violence envers les enfants dans la population québécoise. Revue canadienne de santé publique, 2, 109-113.

Tourigny, M., Hébert, M., Joly, J., Cyr, M., \& Baril, K. (2008). Prevalence and co-occurrence of violence against children in the Quebec population. Australian and New Zealand Journal of Public Health, 32, 331-335.

Trocmé, N., \& Wolfe, D. (2001). Maltraitance des enfants au Canada : Résultats choisis tirés de l'Étude canadienne sur l'incidence des signalements de cas de violence et de négligence envers les enfants. Ottawa : Ministre des Travaux publics et Services gouvernementaux.

Tummala-Narra, P. (2007). Conceptualizing trauma and resilience across diverse contexts: A multicultural perspective. Journal of Aggression, Maltreatment \& Trauma, 14, 33-53.

Ungar, M., \& Liebenberg, L. (2005). The International Resilience Project: A mixed methods approach to the study of resilience across cultures. In M. Ungar (Éd.), Handbook for working with children and youth: Pathways to resilience across cultures and contexts. Thousand Oaks, CA: Sage.

Vallieres, E. F., \& Vallerand, R. J. (1990). Traduction et validation canadienne-française de l'échelle de l'estime de soi de Rosenberg. International Journal of Psychology, 25, 305-316.

Veitch-Wolfe, V., Gentile, C., \& Wolfe, D. A. (1989). The impact of sexual abuse on children: A PTSD formulation. Behavior Therapy, 20, 215-228.

Wolkow, K. E., \& Ferguson , B. H. (2001). Community factors in the development of resiliency: Considerations and future directions. Community Mental Health Journal, 37, 489-498.

Wright, J., \& Sabourin, S. (1996). Traduction du Trauma Symptom Checklist for Children (TSC-C) (Briere, 1989). Document inédit, Université de Montréal.

Yellow Horse Brave Heart, M., \& DeBruyn, L. M. (1998). The American Indian holocaust: Healing historical unresolved grief. The Journal of the National Center, 8, 60-82.

Zahradnik, M., Stewart, S. H., O'Connor, R. M., Stevens, D., Ungar, M., \& Wekerle, C. (2010). Resilience moderates the relationship between exposure to violence and posttraumatic reexperiencing in Mi'kmaq youth. International Journal of Mental Health and Addiction, 8, 408-420. 